\title{
Optimizing pain control through the use of implantable pumps
}

\author{
Wilfried Ilias' \\ Boris Todoroff ${ }^{2}$ \\ 'Dept Anesthesiology, Intensive \\ Care Medicine and Pain Therapy, \\ Academic Teaching Hospital St. John \\ of God,Vienna, Austria; ${ }^{2}$ Dept. Plastic \\ and Reconstructive Surgery, Hospital \\ of St. Vincent, Vienna, Austria
}

\begin{abstract}
Intrathecal therapy represents an effective and well established treatment of nonmalignant as well as malignant pain. Devices available include mechanical constant flow pumps as well as electronic variable flow pumps with patient-controlled bolus release. The latter provide faster dose finding, individual pain control, and good acceptance by patients. New technologies such as membrane pumps and rechargeable devices are expected to be developed to clinical perfection. The available drugs for intrathecal therapy are listed according to the polyanalgesic consensus on intrathecal therapy. The integration of remote patient-controlled analgesia into electronic implantable devices, and the peptide analgesic ziconotide, have significantly improved intrathecal therapy. Complications include infections, catheter ruptures or disconnections, catheter granulomas, and technical dysfunctions. Further possibilities for optimizing intrathecal therapy include development of new drugs, drug side effects, catheter and pump technologies, and surgical techniques.
\end{abstract}

Keywords: intrathecal therapy, implantable pumps, morphine pumps, intrathecal drugs, intrathecal catheters, intrathecal pain control

\section{Introduction}

Pain control is not only a question of an individual's need, but also an increasing socioeconomic issue. It may be presumed that not only pain specialists but also physicians in general are aware that pain meets the definition of illness if it dominates the life of an individual, and interferes with that individual's undisturbed participation in normal life. There are many reports on epidemiology and socio-economic consequences (Elliott et al 1999). According to these data, adequate pain treatment demands both a therapeutic infrastructure (equipment, facilities and staff) as well as the availability of therapeutics.

Spinal application of analgesics offers many advantages. The doses needed to provide pain relief are very low. Because there is almost no systemic distribution of the drugs, side effects are reduced. The first successful attempts to control intractable pain by continuous centroaxial application of morphine started in the late 1970s and early 1980s by means of external infusion systems (Onofrio et al 1981). After successful application of an implantable device (Harbaugh et al 1982), the first FDA-approved implantable pump (Infusaid Model 400; Norwood, MA, USA) was launched. However, this pump and its successors were designed only to deliver a constant flow of drugs. Very soon it became apparent that in patients suffering pain of various levels of intensity and sudden attacks of high intensity, the constant flow technology could not meet the demands, and that this so-called breakthrough pain needed to be controlled by additional systemic drug regimens. Since patient-controlled anesthesia (PCA) had been invented in the mid 1970s and had proven to be very effective in controlling breakthrough pain (Evans et al 1976), efforts were made to develop systems that would enable the constant flow by boluses to be topped up on demand. One example has been the AlgoMed ${ }^{\circledR}$ pump (Medtronic Inc., Minneapolis, 
MN, USA), which works on the basis of a gas-driven, capillarycontrolled constant flow with a deformable reservoir, releasing a bolus when compressed (McMullen et al 1997). A more recent version of this invention is still in use and proved to be very cost effective (Erdine et al 2007). Based on a programmable battery-driven pump for the control of insulin release, the rollerpump-driven devices of the SynchroMed ${ }^{\circledR}$ (Medtronic Inc.) series seem to be the most advanced technology now in clinical use. This system, containing many electronic and mechanical parts, needs valuable space for the pump controller which limits the remaining space available for the drug reservoir. Further innovative designs, with space-sparing membrane pump technology driven by piezoelectric devices, have been invented and successfully tested under clinical conditions (Kan et al 2004) and seem to a promising and space-saving alternative to the roller-pump technology.

The technical demands of implantable systems and also the drugs used for intrathecal or epidural application are being constantly studied. The relative merits of epidural and intrathecal approaches are also under debate. Because of frequent reports of epidural masses after prolonged drug application (Rodan et al 1985), clinicians have favored intrathecal application, but there have been increasing numbers of reports of inflammatory masses surrounding the catheter tips with this approach (Bennett et al 2000; Coffey and Burchiel 2002; Hassenbusch et al 2004). It became clear from these reports that the incidence of catheter granulomas increased when the concentration of morphine exceeded $1.5 \%$ or $15 \mathrm{mg} / \mathrm{mL}$ of the solution. However, owing to differences in underlying pain mechanisms and individual sensitivity to morphine and/or other analgesics, and the limited volume of the implanted device, flow rates will be reflected in decreasing refill intervals. It should be pointed out that the only way to increase the refill interval of the implanted device is to increase the concentration of the solution. It is well known that there is an increasing risk of neurotoxicity related to drug concentration, osmolarity, and local distribution in the spinal fluid (Malinovsky and Pinaud 1996; Auroy et al 1997; Gerancher 1997; Holman et al 1997; Chan et al 1998; Holst et al 1998; Loo and Irestedt 1999). In order to overcome these incompatibilities or risks, several drugs, combinations, and concentrations have been experimentally tested.

Medical procedures or regimens need to be optimized or improved when the available options show insufficient effectiveness, a need of extraordinary skills, or are of considerable risk. In the following sections the main requirements for optimizing intrathecal therapy are defined by breaking down intrathecal therapy in to its component parts. The available drugs still show deficiencies in effectiveness, stability in surrounding body temperature, compatibility of certain concentrations, lack of neural toxicity, as well as adverse side effects. Thus there is an ongoing effort to develop new drugs that will better meet these demands, either in single use or in combinations.

\section{Optimizing drug regimes}

A recent overview of drugs and combinations for intrathecal pain treatment is given in the recommendation of the Polyanalgesic Consensus Conference 2007 (Deer et al 2007). Contrary to the recommendations of Hassenbusch et al (2004), the same panel, because of new evidence, listed ziconotide as well as morphine and hydromorphone as firstline in their algorithm for intrathecal polyanalgesic therapies (Deer et al 2007). The decision was based on the fact that morphine and ziconotide (Prialt ${ }^{\circledR}$, Elan Pharmaceuticals, Inc.; Eisai Europe Ltd) are FDA approved for intrathecal analgesic use, and because both drugs are effective in nociceptive, mixed, and neuropathic pain. Hydromorphone has also been recommended as a first-line drug in this algorithm because of widespread use and apparent safety. For the same reason, and also because of its granuloma-sparing effect, fentanyl has been upgraded to a second-line drug together with mixtures of morphine/hydromorphone + ziconotide and morphine/hydromorphone + bupivacaine/clonidine. Clonidine as a single drug and morphine/hydromorphone/ fentanyl + bupivacaine/clonidine + ziconotide are third-line drugs. Sufentanil, which also has apparent granuloma-sparing effect and is increasing in use, was ranked a fourth-line choice. Deer et al found that sufentanil may be used also in combination with bupivacaine, clonidine, or ziconotide. Further drugs, listed by this panel as fifth- and sixth-line choices, are not mentioned here because the consensus panel thought that there was too little information in the literature and their use can be recommended only with caution. One of these drugs is the opioid buprenorphine. It is mentioned here because there are persuasive reports on its feasibility for intrathecal treatment of intractable pain (Nitescu et al 1998), even for long-term treatment of up to 6 years (Dahm et al 1998). This opioid has been used solely in concentrations of 0.015 to $0.017 \mathrm{mg} / \mathrm{mL}$ or mixed with the local anesthetic bupivacaine $4.75 \mathrm{mg} / \mathrm{mL}$. Daily doses ranged from 0.02 to $0.2 \mathrm{mg}$ buprenorphine and 10 to $70 \mathrm{mg}$ bupivacaine (Dahm et al 1999). In this context, it is important to note that the panelists stated, "clinical research in intrathecal analgesics, that meets the gold standard of evidence-based studies, has not kept pace with the growing need for innovative 
approaches to pain management" (Deer et al 2007). Another important point in choosing a certain drug or combination may be the fact that exposure of a drug in a device made of metal and biologically compatible tubes under a surrounding temperature of more than $37^{\circ} \mathrm{C}$ may influence the stability of a certain drug or mixtures, as mentioned above. The usual tests, however, refer only to the possible degradation of a certain molecule but not to the biological activity of a drug. In larger molecules, such as ziconotide, which in chemical testing showed degradation when exposed to $37^{\circ} \mathrm{C}$ and when mixed with morphine (Shields and Montenegro 2007), it is yet clear whether this degradation affects its biological activityt. No reports are available on decreasing analgesia in ziconotide-treated patients during usual pump-refill intervals of 4 to 6 weeks. Hence it would be worthwhile to check whether drugs and solutions, which in chemical set ups show degradation of the original molecules such as ziconotide, also have different activity when tested in biological receptors.

The implantable pumps available for clinical use, although of very advanced technology, certainly need further adaptations in order to meet the demands of therapeutic flexibility and overall acceptance.

\section{Optimizing pain control by pump technology innovations}

The control of unpredictable pain fluctuations has been a challenge to intrathecal therapy because, as mentioned above, the first generations of implantable pumps were designed only to provide a constant flow of drugs. Although attempts to integrate bolus functions into gas-driven constant flow devices were successful (McMullen et al 1997), programmable infusion pumps are superior not only for individualized infusion programs in spasticity and pain (Penn and Kroin 1987), but also for individual adaptation of intraperitoneal insulin application in diabetic patients (Saudek and Selam 1989). Compared with constant-flow devices, programmable pumps offer many advantages:

If pain levels or the sensitivity to the drug in use changes, increasing or decreasing demands can be easily met by changing the speed of infusion with a remote control device whenever necessary. To achieve this change in constant-flow devices the concentration of the drug solution needs to be changed. This presumes repeated emptying and refilling of the pump reservoir through a puncture hole. Good experience in estimating individual demands are necessary in order to avoid short-interval repetitions of this procedure.

Predictive changes in pain levels owing to physical and emotional stress or resting periods as well as other individual circadian rhythms can now be controlled by adapting personal infusion schemes to individual demands via remote programming. The same is possible with fluctuating spasticity after reprogramming baclofen treatment, or with fluctuating blood glucose levels after food intake and insulin infusion.

Despite technical progress in pump design, clinical practice shows that many patients, even though under treatment with individually adapted infusion schemes, lack adequate pain relief because of non-predictive and, in part, sudden changes in pain intensity. Further improvements in implantable pumps were inevitable, in terms of the individualization of therapy, because the advantages of PCA provided by external devices was already well established and also known to be very effective. In a comprehensive review of data proving the existence and severity of not only predictable but also unpredictable fluctuations of pain intensity and spasticity, we concluded that even programmable pumps cannot be used reliably to administer the appropriate amount of medication at any particular time, and that a combination of PCA and programmable drug delivery should be integrated in the next generation of programmable devices (Rainov et al 2002). The first device in clinical use to meet these demands (SynchroMed $\mathrm{EL}^{\circledR}$ ) allows the patient to recall bolus infusion in advance of expected increases in pain (prophylactic) or in response to sudden breakthrough attacks (therapeutic). As in the usual external PCA devices, the dose, infusion time, lock-out interval, and maximum repetitions of boluses per day are programmable. Each bolus recalled will be counted and registered as successful, rejected, or unsuccessful (due to handling errors). This event registration is integrated in the pump memory and allows the physician to estimate, from readouts, the real demands and to re-adapt when necessary both the basic infusion dose and the bolus dose. If the registration of events shows many unsuccessful communications, the doctor can easily deduce whether the patient has handling problems and needs further instruction with the device. The next generations (SynchroMed II and PTM = Patient Therapy Manager) of programmable, implantable pumps with patient-therapy-managing remote control now have additional useful features such as provision of the option for the patient to register the value of a visual analog pain scale (VAS) before recalling a bolus, and to do so after a defined interval after the bolus. In order to remind the patient, the PTM will sound an alarm and ask for VAS input. All values are stored, and again the physician is able to analyze bolus counts and bolus responses and to adapt the pump's infusion scheme to the individual demands. These programmable pumps can also calculate and predict refill 
intervals according to the sum of volumes of the constant infusion and the maximum of possible boluses. And, of course, by sounding an alarm, the implanted device will remind the patient when the container volume falls below a predefined alarm value. If the patient needs more time to reach the refilling institution, it may be worthwhile to take this into consideration when predefining the volume alarm limit. Finally, if not all possible boluses are used, the PTM will recalculate the refill time according to the remaining volume and display the time on the screen, thereby allowing patients to increase and control refill interval according to their individual demands. Of course, these many technical refinements are expensive and raise the question of whether they are cost effective. A multi-center study, which included 168 patients with chronic cancer and non-cancer pain treated by intrathecal drug application by means of programmable pumps and the above-mentioned PTMs, showed a very good acceptance of the device after a 12 -month period. The patients were able to manage unpredictable pain fluctuations very actively, easily, and safely, and also learned to use the device to control expected pain fluctuations by prophylactic recall of boluses. Repeated bolus and VAS readouts after device implantation devices shortened the time needed for physicians to optimize basal rates of drug delivery according to individual demands. On the other hand, optimal dose range was reflected by a decrease in average daily bolus recalls. Overall satisfaction and acceptance of the PTM by patients was greater than $80 \%$. The doctors reported better analgesia, individualized treatment, and patient independence. Doctors and patients preferred a smaller PTM for easier handling, and both found that the time needed to understand how to use and handle the device was too long (Ilias et al 2008).

Programmable pumps, because of their electronic and mechanical technology, are very bulky and currently offer far less volume capacity than constant-flow devices of similar external diameter. Depending on infusion speed necessary to deliver the demanded dose, batteries may be exhausted long before their expected lifetime, which happens when the drug solutions reach their maximum recommended concentrations (Deer et al 2007) and the daily doses necessary to provide analgesia exceed a volume of more than 1 to $2 \mathrm{~mL}$. Hence there is also a need for new pump technologies needing less space and energy. Membrane pumps driven by piezo-electric mini crystals seem to meet both demands compared with other electronic pumps currently in clinical use (Kan et al 2004). Rechargeable devices for spinal cord stimulation are now being developed by Advanced Neuromodulation Systems (ANS), Boston Scientific, and Medtronic. This technology could also be applied to intrathecal pumps, but no information is available on whether or how this technology is being adopted in medicine. Remote magnetic control could be used to open and close electronic capillaries, which could be used in gasdriven pumps, thereby combining electronic advantages with space-saving technology. Patents are pending on various designs. Again, no studies are available to prove the feasibility of these technologies. Ideas to split the drug reservoirs from the steering (or control) part are not new and have been incorporated in an elastomer and capillary flow directed device with a patient control panel (AlgoMed ${ }^{\circledR}$; Medtronic Inc.), which is still in clinical use and apparently is highly cost effective (Erdine and Talu 2007). The advantage of such devices is that the steering part and the refill port can be integrated in a protective and robust shell, with a flat design in a very small set up. The reservoir can be designed as a robust but flexible vessel, made of a biologically compatible and stress-resistant material, which therefore adapts to movements and pressure but does not directly compress or irritate the surrounding tissue, thereby forcing transdermal movement of the implant. Of course if such a vessel containing extraordinarily high doses of certain analgesics or mixtures of analgesics bursts due to extraordinary physical stress, or is accidentally injured by needles or other sharp instruments, the whole contents will be distributed into the tissue pocket and eventually transported through the bloodstream. The result will be a dramatic and harmful systemic reaction, the extent of which will depend on the reservoir content and the type of drug or mixture. Whereas most of the line 1 to 4 drugs mentioned in the polyanalgesic consensus (Deer et al 2007) may be of high risk in such a situation, ziconotide administered intrathecally in humans has not been reported to induce severe adverse reactions. Our own experience with accidental overflow of paravasates of ziconotide during refill procedures had no detectable clinical side effects.

\section{Optimizing catheter technology}

Depending on the materials used, inner and outer diameters of spinal catheters is use vary widely. When placed in the body the spinal and the proximal catheter segments create different physical stresses; hence available catheters have thinner-walled spinal segments and thicker-walled, more robust proximal segments, eg, Medtronic 8731SC. In this model the inner diameter stays constant, and hence the volume of the catheter is defined as $\mu \mathrm{L} / \mathrm{cm}$ of length. Other two-piece catheter models are available, eg, Tricumed Spinal-Katheterset 4000 (Tricumed Medizintechnik GmbH, Kiel, Germany) with different outer diameters (13 and 16.8 G), and also different inner diameters 
(0.7 and $0.6 \mathrm{~mm})$. Usually, when catheters are implanted, they are cut to individual optimal length of distal and proximal segments depending on the height of the spinal segment and the distance from the spinal puncture site to the connector of the pump. In the Medtronic 8731SC model, the volume of the catheter can be calculated by simply adding the lengths of both catheter pieces. The Tricumed Spinal-Katheterset 4000 has different length/volume ratios, requiring an additional calculation. Although this would be easy for academically trained staff, clinical practice proves the truth of the "make it simple" principle. Especially when changing doses or concentrations, or the drug itself, knowledge of the exact catheter volume is of great importance. To enable continuation of therapy, in electronic pumps bridging boluses may be programmed to adapt pump flow rate from the old to the new drug regimen. In constant flow pumps, the catheter contents need to be removed by aspirating the catheter content through the side port and refilling the catheter with the new drug mixture. Because drug concentrations are adapted to very low flows, overestimating catheter length will result in a sudden overdose, and underestimating catheter length in a discontinuation of drug flow, until the solution reaches the end of the catheter.

\section{Complications and how to avoid them}

Although catheter connections are designed to remain connected, disconnections can occur, particularly as a result of the common tissue stresses associated with bodily movements of daily life (Figure 1). In this case (Figure 1), no extraordinary stresses through sports activity, trauma, or other extreme movements were reported. Therefore the patient, although well instructed on all possibilities of (known) complications, including catheter ruptures and pump failures, had no reason to contact the responsible institution. Several days elapsed before the patient realized something was wrong with the implanted device and contacted the responsible physician. Because of this delay, the patient presented not only with severe pain, but also with intense withdrawal symptoms, demanding immediate hospitalization. In order to avoid further risk of disconnection, during the repair surgery the interlocking was not only protected by ligatures but also by medical silicone glue. As a rationale, in order to optimize invasive pain treatment, devices should be designed to decrease further the risk of possible disconnections by incorporating connection designs that provide stress resistance to the catheters themselves.

As mentioned above, hard-cover pumps with metal shells exert a certain stress to the surrounding tissue. Of course,

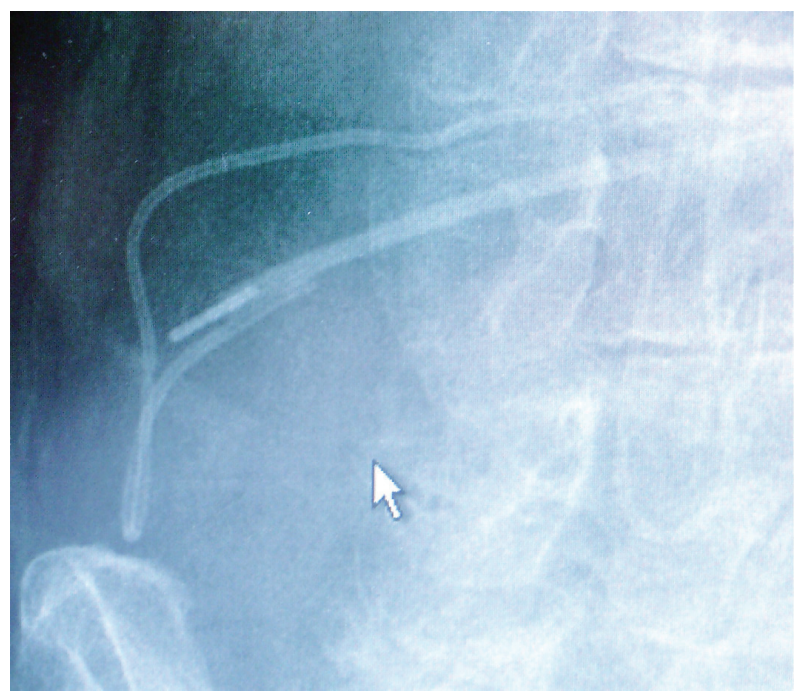

Figure I X-ray control of a suspected catheter rupture. The disconnected catheter endings are clearly visible showing also the metal connector situated in the proximal ending of the catheter.

design of soft-cover reservoir pumps with hard-cover electronic steering parts, thereby dividing the total volume of the device between soft and hard components, would be an advantage. However, a reservoir made of soft materials is less shock resistant than one made of hard materials, at least with current technology. Hard-shell metal pumps, because of the total volume and hardness of the device, may induce tissue necroses, with the risk of infection and loss of the (expensive) device. Our own experience suggests that anatomical factors, such as thin subcutaneous fatty tissue, small abdominal area (in small people), or atrophic skin (cortisone skin), increase the risk of pump bed necrosis. A typical situation is shown in Figure 2. A diabetic, lean patient with a short trunk and resulting small abdominal area developed skin necrosis at the lower edge of the implanted pump. A more median placement did not improve the situation. Therefore, with the assistance of a plastic surgeon the electronic, $40-\mathrm{mL}$ device was explanted and replaced in the fascial sheath of the rectus abdominis muscle (see also Figure 3). When discussing possibilities for optimizing intrathecal therapy, it is worth noting the complete lack of controlled studies on implantation techniques. There is only a recommendation for electronic devices that tissue layers between the device and the skin surface should not exceed $20 \mathrm{~mm}$ in order to provide undisturbed remote control of the device.

\section{Discussion}

Intrathecal therapy has been proven to be very effective in the treatment of chronic therapy-resistant pain. 


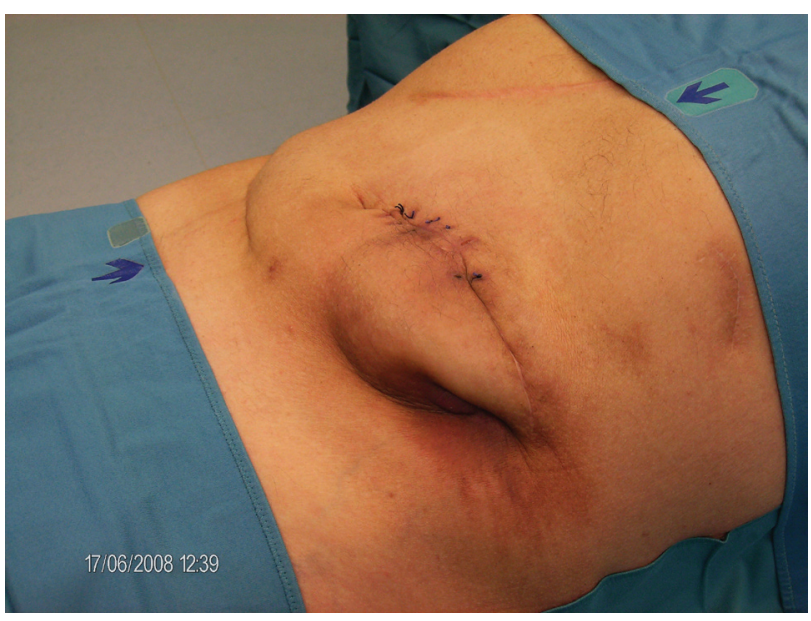

Figure 2 Subcutaneous tissue necrosis at the caudal edge of an implanted SynchroMed II pump. Skin sutures after an attempt to correct the pump placement by moving the device more medially are still visible.

Despite innovations such as electronic, programmable pumps with the potential for patient-controlled bolus application, as well as new drugs, the shortcomings in available therapy remain considerable. These shortcomings are reflected in complications related to intrathecal therapy such as catheter disconnections, spinal fluid leakage, tissue necrosis, battery exhaustion, misses of refill ports with resulting paravasates, drug incompatibilities, catheter granulomas, and seromas around the implants. These complications suggest many possibilities to further optimize intrathecal pain therapy: improving catheter connections to withstand average physical stress; designing catheters that avoid spinal fluid leakage

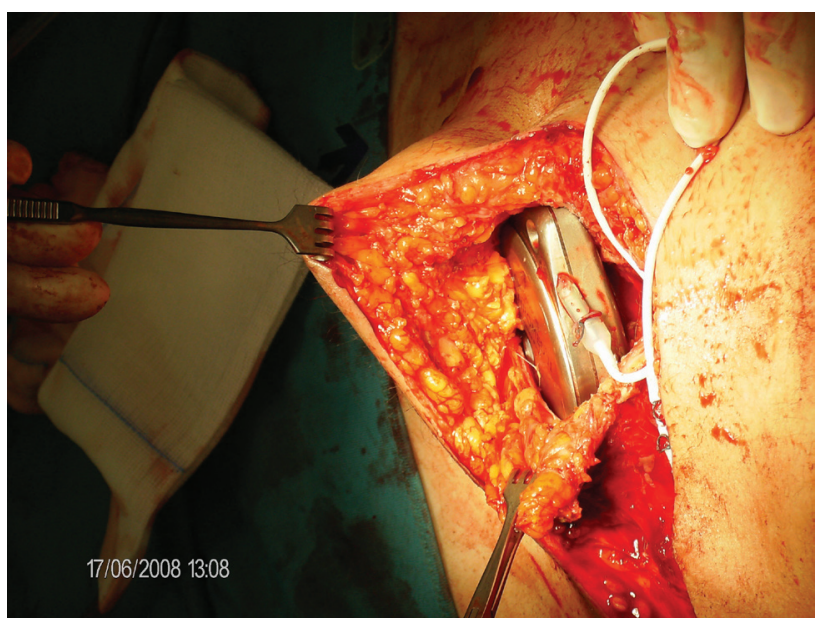

Figure 3 The SynchroMed II pump is now placed in the sheath of the rectus abdominis muscle and fixed to the fascia by non-resorptive sutures. This technique is possible only if the fatty tissue between the skin and electronic device does not exceed $25 \mathrm{~mm}$ in order to provide undisturbed telemetry. along the puncture site, and that resist degradation under exposure to body temperature and non-biological surfaces; to standardize catheter sizes and connectors; to improve implantation techniques; and to find drugs compatible in mixtures. Pesentations on implantation techniques at workshops and expert meetings suggest that catheters may be inserted with or without prior skin cut and subcutaneous preparation of the optimal interlaminar approach. The latter certainly offers the advantage of better anatomical overview and catheter fixation in deeper levels of the tissue, even below muscle. This technique may prevent pain arising from compression of the fixation site and constant tissue stress, which result in transcutaneous migration of the catheter especially in frail individuals. Clear preparation of fascia and ligaments allow catheter fixation to this sort of tissue, thereby providing better protection against dislocation by mechanical stress. No data compare advantages or disadvantages of one or the other technique, but it may be suggested that experienced implantation centers with standardized surgical implantation procedures will have lower complication rates (Koulousakis et al 2007) and provide expertize such as neurosurgical backup. In case of complications such as spinal fluid leakages, meticulous surgical skills are demanded (Singh et al 2008).

\section{Disclosures}

The authors have no conflicts of interest to disclose.

\section{References}

Auroy Y, Narchi P, et al. 1997. Serious complications related to regional anesthesia: results of a prospective survey in France. Anesthesiology, 87:479-86.

Bennett G, Burchiel K, et al. 2000. Clinical guidelines for intraspinal infusion: report of an expert panel. PolyAnalgesic Consensus Conference 2000. J Pain Symptom Manage, 20:S37-43.

Chan VW, Garcia J, et al. 1998. A comparative study of low-dose hyperbaric spinal lidocaine $0.5 \%$ versus $5 \%$ for continuous spinal anesthesia. Reg Anesth Pain Med, 23:164-9.

Coffey RJ, Burchiel K. 2002. Inflammatory mass lesions associated with intrathecal drug infusion catheters: report and observations on 41 patients. Neurosurgery, 50:78-86; discussion 86-7.

Dahm PO, Nitescu PV, et al. 1998. Six years of continuous intrathecal infusion of opioid and bupivacaine in the treatment of refractory pain due to intrapelvic extrusion of bone cement after total hip arthroplasty. Reg Anesth Pain Med, 23:315-9.

Dahm PO, Nitescu PV, et al. 1999. Intrathecal infusion of bupivacaine with or without buprenorphine relieved intractable pain in three patients with vertebral compression fractures caused by osteoporosis. Reg Anesth Pain Med, 24:352-7.

Deer T, Krames ES, et al. Hassenbusch S, et al. 2007. Polyanalgesic Consensus Conference 2007: Recommendations for the Management of Pain by Intrathecal (Intraspinal) Drug Delivery: Report of an Interdisciplinary Expert Panel. Neuromodulation, 10:300-28.

Elliott A, Smith BH, et al. 1999. The epidemiology of chronic pain in the community. Lancet, 354:1248-52.

Erdine ST, Talu GK. 2007. Cost effectiveness of implantable devices versus tunneled catheters. Current Pain Headache Rep, 2:157-62. 
Evans JM, Rosen M, et al. 1976. Apparatus for patient-controlled administration of intravenous narcotics during labour. Lancet, 1(7949):17-18.

Gerancher JC. 1997. Cauda equina syndrome following a single spinal administration of $5 \%$ hyperbaric lidocaine through a 25 -gauge Whitacre needle. Anesthesiology, 87:687-9.

Harbaugh RE, Coombs DW, et al. 1982. Implanted continuous epidural morphine infusion system. Preliminary report. J Neurosurg, 56:803-6.

Hassenbusch SJ, Portenoy RK, et al. 2004. Polyanalgesic Consensus Conference 2003: an update on the management of pain by intraspinal drug delivery - report of an expert panel. J Pain Symptom Manage, 27:540-63.

Holman SJ, Robinson RA, et al. 1997. Hyperbaric dye solution distribution characteristics after pencil-point needle injection in a spinal cord model. Anesthesiology, 86:966-73.

Holst D, Mollmann M, et al. 1998. Intrathecal local anesthetic distribution with the new spinocath catheter. Reg Anesth Pain Med, 23:463-8.

Ilias W, Le Polain B, et al. 2008. Patient-controlled analgesia in chronic pain patients: experience with a new device designed to be used with implanted programmable pumps. Pain Practice, 8:164-70.

Kan J, Yang Z, et al. 2004. [Pumping performance of a new piezoelectric pump for drug delivery]. Sheng Wu Yi Xue Gong Cheng Xue Za Zhi, 21:297-301.

Koulousakis A, Kuchta J, et al. 2007. Intrathecal opioids for intractable pain syndromes. Acta Neurochir Suppl, 97:43-8.

Loo CC, Irestedt L. 1999. Cauda equina syndrome after spinal anaesthesia with hyperbaric $5 \%$ lignocaine: a review of six cases of cauda equina syndrome reported to the Swedish Pharmaceutical Insurance 1993-1997. Acta Anaesthesiol Scand, 43:371-9.
Malinovsky JM, Pinaud M. 1996. [Neurotoxicity of intrathecally administrated agents]. Ann Fr Anesth Reanim, 15:647-58.

McMullen R, Kratoska P, Gardner A. 1997. Infusion Systems with Patient-Controlled Dosage Features. Patent Storm. I. MedTronic. 9723252 WO.

Nitescu,P, Dahm P, et al. 1998. Continuous infusion of opioid and bupivacaine by externalized intrathecal catheters in long-term treatment of "refractory" nonmalignant pain. Clin J Pain, 14:17-28.

Onofrio BM, Yaksh TL, Arnold PG. 1981. Continuous low-dose intrathecal morphine administration in the treatment of chronic pain of malignant origin. Mayo Clin Proc, 56:516-20.

Penn RD, Kroin JS. 1987. Long-term intrathecal baclofen infusion for treatment of spasticity. J Neurosurg, 66:181-5.

Rainov NG, Buchser E. 2002. Making a case for programmable pumps over fixed rate punps for the management of fluctuations in chronic pain and spasticity: a literature review. Neuromodulation, 5: 89-99.

Rodan BA, Cohen FL, et al. 1985. Fibrous mass complicating epidural morphine infusion. Neurosurgery, 16:68-70.

Saudek CS, Selam JL. 1989. A preliminary trial of the programmable implantable medication system for insulin delivery. N Engl J Med, 321:574-9.

Shields D, Montenegro R. 2007. Chemical stability of ziconotideclonidine hydrochloride admixtures with and without morphine sulfate during simulated intrathecal administration. Neuromodulation, 10(S 1):6-11.

Singh PK, Jain R, et al. 2008. Management of pericatheter cerebrospinal fluid leak after intrathecal implantation of a drug delivery system. Am J Hosp Palliat Care, 25:237-9. 
